\title{
The Characteristics of Solar X-Class Flares and CMEs: A Paradigm for Stellar Superflares and Eruptions?
}

\author{
Louise K. Harra ${ }^{1}$ (D) - Carolus J. Schrijver ${ }^{2} \cdot$ Miho Janvier $^{3} \cdot$ Shin Toriumi $^{4}$. \\ Hugh Hudson $^{5}$ - Sarah Matthews ${ }^{1}$ - Magnus M. Woods ${ }^{1}$. Hirohisa Hara ${ }^{4}$. \\ Manuel Guedel $^{6}$ - Adam Kowalski ${ }^{7}$ - Rachel Osten ${ }^{8}$ - Kanya Kusano ${ }^{9}$. \\ Theresa Lueftinger 6
}

Received: 22 December 2015 / Accepted: 22 May 2016 / Published online: 27 June 2016

(C) The Author(s) 2016. This article is published with open access at Springerlink.com

\begin{abstract}
This paper explores the characteristics of 42 solar X-class flares that were observed between February 2011 and November 2014, with data from the Solar Dynamics Observatory (SDO) and other sources. This flare list includes nine X-class flares that had no associated CMEs. In particular our aim was to determine whether a clear signature could be identified to differentiate powerful flares that have coronal mass ejections (CMEs) from those that do not. Part of the motivation for this study is the characterization of the solar paradigm for flare/CME occurrence as a possible guide to the stellar observations; hence we emphasize spectroscopic signatures. To do this we ask the following questions: Do all eruptive flares have long durations? Do CME-related flares stand out in terms of active-region size vs. flare duration? Do flare magnitudes correlate with sunspot areas, and, if so, are eruptive events distinguished? Is the occurrence of CMEs related to the fraction of the activeregion area involved? Do X-class flares with no eruptions have weaker non-thermal signatures? Is the temperature dependence of evaporation different in eruptive and non-eruptive flares? Is EUV dimming only seen in eruptive flares? We find only one feature consistently associated with CME-related flares specifically: coronal dimming in lines characteristic of
\end{abstract}

$凶$ L.K. Harra

I.harra@ucl.ac.uk

1 UCL-Mullard Space Science Laboratory, Holmbury St Mary, Dorking, Surrey, RH5 6NT, UK

2 Lockheed Martin Solar and Astrophysics Laboratory, 3251 Hanover Street, Palo Alto, CA 94304, USA

3 Institut d'Astrophysique Spatiale, UMR8617, Univ. Paris-Sud-CNRS, Université Paris-Saclay, Bâtiment 121, 91405 Orsay Cedex, France

4 National Astronomical Observatory of Japan, 2-21-1 Osawa, Mitaka, Tokyo 181-8588, Japan

5 SUPA School of Physics and Astronomy, University of Glasgow, Glasgow, UK

6 Department of Astrophysics, University of Vienna, Türkenschanzst. 17, 1180 Vienna, Austria

7 Department of Astronomy, University of Maryland, College Park, MD, 20742, USA

8 Space Telescope Science Institute, 3700 San Martin Drive, Baltimore, MD 21218, USA

9 Solar-Terrestrial Environment Laboratory, Nagoya University, Furo-cho, Chikusa-ku, Nagoya, Aichi 464-8601, Japan 
the quiet-Sun corona, i.e. 1-2 MK. We do not find a correlation between flare magnitude and sunspot areas. Although challenging, it will be of importance to model dimming for stellar cases and make suitable future plans for observations in the appropriate wavelength range in order to identify stellar CMEs consistently.

Keywords Corona $\cdot$ Flares, dynamics $\cdot$ Flares, magnetic fields

\section{Introduction}

Solar flares are among the most energetic phenomena in our solar system, and there continues to be a large international effort to understand the physical processes that release such vast amounts of energies in minutes. Coronal mass ejections (CMEs), which are often associated with solar flares, have comparable energies, and can release large amounts of mass (up to $10^{16} \mathrm{~g}$ ) into the heliosphere. These ejecta and associated high-energy particles may strongly affect planetary environments (e.g. Gosling et al., 1991)

Over the years, the ever-improving observations have helped provide evidence (or otherwise) for a solar CME-associated flare model which assumes a twisted magnetic structure or flux rope rising in the corona, stressing the surrounding field lines and causing magnetic reconnection to occur; this then would heat the local coronal plasma and accelerate the particles. This model as developed by Carmichael (1964), Sturrock (1966), Hirayama (1974) and Kopp and Pneuman (1976) (hence CSHKP for short) appeared in the 1960s and 1970s and has since been extended. The thick-target model of electron transport then hypothesizes particle heating of the chromospheric footpoints, leading to the observed "evaporation" of plasma into the reconnected magnetic field and "condensation" of material toward the footpoints. The heated plasma that rises into the corona radiates predominantly in the XUV spectral range, with temperatures of 10-20 MK, much hotter than the chromospheric temperatures at which the majority of the energy is emitted. These coronal flare loops then cool down and become visible in the lower temperature emissions (see e.g. Forbes and Acton, 1996; Tsuneta, 1996).

Many observational features match this CSHKP scenario: the flare ribbons separate as reconnection occurs higher in the atmosphere, evaporation (up-welling blue-shifted plasma) occurs in the ribbons, non-thermal particles appear at footpoint regions within the ribbons (see e.g. Fletcher et al., 2011 and references therein). Finally, recent numerical simulations have helped to adapt this standard model more fully to three dimensions (3D), and to describe some of its observational features, such as the change of shear in subsequent generations of cooling flare loops and the shape of flare ribbons (see e.g. Janvier et al. (2014) and references therein).

In this paper we study the 42 solar X-class flares that were observed between February 2011 and November 2014, basically to ask several questions that may lead to a systematic understanding of their properties, and especially to provide a modern view of the solar paradigms that might be applied to similar activity in other stars. We would like to be able to identify the presence or absence of a CME from "Sun-as-a-star" observations, noting that stellar observations of CMEs are difficult (Leitzinger et al., 2014) but important for the question of exoplanet habitability and long-term stellar angular momentum loss. This event list generally has the best observational database available, since it is recent, and the X-class flares are the most powerful solar events, approaching in terms of energy the low end of the energy range for which stellar flares are detectable. 
At present, there is much discussion relating the morphology of flares and CMEs to emerging flux, sheared fields, magnetic cancellation, interacting coronal loops, sigmoid structures, etc., but all of these properties tend to be qualitative in nature. As such, the aim of the present study is to question the possibility of a statistical study on the largest flares recorded at high spatial and temporal resolution with the Solar Dynamics Observatory (SDO; Pesnell, Thompson, and Chamberlin, 2012) to provide more clues to constrain a generic model. In this overview, we suggest the possibility of two categories of flares: eruptive flares and non-eruptive flares. This softens the category of "compact" flares (Pallavicini, Serio, and Vaiana, 1977), because we have learned over the past decades that non-eruptive flares do not need to be physically compact - and eruptive flares may be. We also avoid the wording "confined eruptions" as this does not distinguish flares that do not display evidence of the presence of a flux rope, either during the flare or prior to the flare (e.g. sigmoids) from "failed eruptions", for which the motion of rising prominences and filaments appears to stall, with material subsequently falling back to the Sun instead of erupting into the heliosphere (Ji et al., 2003). These failed eruptions have no CME association, but they tend to have the other properties of an eruptive flare. It is difficult to predict whether a flare will be confined or eruptive from the observations, and so the exploration of this issue is a major objective of this study. Schrijver (2009) provides a review of what drives flares, associated with flux-rope signatures, and with or without CMEs. This review highlights two apparently dominating aspects of flare energy conversion that appear to dictate whether or not they are confined or can erupt freely. The first important factor is that of the internal twist of the emerging flux rope. The next factor is that of the properties of the embedding and surrounding magnetic field. To understand these factors in detail could benefit from detailed modeling of each individual event, but this is far beyond the scope of the discussion here and may in most cases be premature, given the limitations of the observational material.

\section{Flares, CMEs, and X-Class Flares Without CMEs}

Generally CMEs represent the heliospheric manifestations of magnetic energy conversion at the Sun, and flares the radiation effects. These phenomena often appear simultaneously, especially for major events (the X-class flares). It is unusual, but not unheard of, for X-class flares to have no associated CME; our list of $42 \mathrm{X}$-class flares (Table 1) contains nine such events (note that six of these were from a single active region). The X-class flare SOL201111-03 is a good example of a major flare without a CME (Liu et al., 2014). The event was compact in low-temperature spectral bands of Atmospheric Imaging Assembly (AIA; Lemen et al., 2012) on SDO, and showed a cusp-shaped feature in the high-temperature coronal bands. Such a cusp, in an eruptive flare, would provide evidence of ongoing magnetic reconnection in the current sheet formed in the aftermath of a flux-rope expulsion, but in this case it requires a different interpretation. There are two arcades involved in the event, one of which has a filament that does not erupt - possibly due to the strong confining field - and a long-duration flare. These properties also usually point to the standard flare model of an eruptive flare, with reconnection continuing to extract energy from the field and cause the formation of the arcade of flare loops. In this case, Liu et al. (2014) suggested that new emergence of magnetic field in the region of the second arcade triggered the events.

More recently a remarkable active region in October 2014, NOAA 12192, produced a series of X-class flares that were non-eruptive. Thalmann et al. (2015) analyzed these events, and found, by using global magnetic field modeling, the existence of a strong north-south large-scale magnetic field that could serve to confine the flare process to the core of the active 
Table 1 The X-class flare sample.

\begin{tabular}{|c|c|c|c|c|c|c|}
\hline IAU & GOES & Location & $\mathrm{AR}$ & Area $^{\mathrm{a}}$ & Eruptive? & SEP? \\
\hline SOL2011-02-15T01:56 & $\mathrm{X} 2.2$ & S20W10 & 1158 & 450 & Yes & Yes \\
\hline SOL2011-03-09T23:23 & $\mathrm{X} 1.5$ & N08W09 & 1166 & 550 & No & No \\
\hline SOL2011-08-09T08:05 & X6.9 & N17W69 & 1263 & 450 & Yes & Yes \\
\hline SOL2011-09-06T22:20 & $\mathrm{X} 2.1$ & N14W18 & 1283 & 220 & Yes & Yes \\
\hline SOL2011-09-07T22:38 & $\mathrm{X} 1.8$ & N14W28 & 1283 & 200 & Yes & Yes \\
\hline SOL2011-09-22T11:01 & $\mathrm{X} 1.4$ & N11E60 & 1302 & 480 & Yes & Yes \\
\hline SOL2011-09-24T09:40 & $\mathrm{X} 1.9$ & N12E60 & 1302 & 840 & Yes & Yes \\
\hline SOL2011-11-03T20:27 & $\mathrm{X} 1.9$ & N22E63 & 1339 & 560 & No & No \\
\hline SOL2012-01-27T18:37 & $\mathrm{X} 1.7$ & N27W71 & 1402 & 270 & Yes & Yes \\
\hline SOL2012-03-05T04:09 & $\mathrm{X} 1.1$ & N17E52 & 1429 & 700 & Yes & Yes \\
\hline SOL2012-03-07T00:24 & $\mathrm{X} 5.4$ & N17E15 & 1429 & 1120 & Yes & Yes \\
\hline SOL2012-03-07T01:14 & $\mathrm{X} 1.3$ & $\mathrm{~N} 22 \mathrm{E} 12$ & 1430 & 1120 & Yes & Yes \\
\hline SOL2012-07-06T23:08 & $\mathrm{X} 1.1$ & S13W59 & 1515 & 670 & Yes & Yes \\
\hline SOL2012-07-12T16:49 & $\mathrm{X} 1.4$ & S15W01 & 1520 & 1320 & Yes & Yes \\
\hline SOL2012-10-23T03:17 & $\mathrm{X} 1.8$ & S10E42 & 1598 & 370 & No & No \\
\hline SOL2013-05-13T02:17 & $\mathrm{X} 1.7$ & N12E67 & 1748 & 250 & Yes & Yes \\
\hline SOL2013-05-13T16:05 & $\mathrm{X} 2.8$ & N11E85 & 1748 & 250 & Yes & Yes \\
\hline SOL2013-05-14T01:11 & $\mathrm{X} 3.2$ & N12E67 & 1748 & 250 & Yes & No \\
\hline SOL2013-05-15T01:48 & $\mathrm{X} 1.2$ & N12E64 & 1748 & 310 & Yes & Yes \\
\hline SOL2013-10-25T08:01 & $\mathrm{X} 1.7$ & S08E59 & 1882 & 100 & Yes & Yes \\
\hline SOL2013-10-25T15:03 & $\mathrm{X} 2.1$ & S08E59 & 1882 & 100 & Yes & Yes \\
\hline SOL2013-10-28T02:03 & $\mathrm{X} 1.0$ & N04W66 & 1875 & 790 & Yes & Yes \\
\hline SOL2013-10-29T21:54 & $\mathrm{X} 2.3$ & N06W91 & 1875 & 770 & Yes & No \\
\hline SOL2013-11-05T22:12 & $\mathrm{X} 3.3$ & S12E46 & 1890 & 830 & Yes & No \\
\hline SOL2013-11-08T04:26 & $\mathrm{X} 1.1$ & S14E15 & 1890 & 920 & Yes & No \\
\hline SOL2013-11-10T05:14 & $\mathrm{X} 1.1$ & S14W13 & 1890 & 660 & Yes & No \\
\hline SOL2013-11-19T10:26 & $\mathrm{X} 1.0$ & S13W79 & 1893 & 480 & Yes & Yes \\
\hline SOL2014-01-07T18:32 & $\mathrm{X} 1.2$ & S12W08 & 1944 & 1415 & Yes & Yes \\
\hline SOL2014-02-25T00:49 & $\mathrm{X} 4.9$ & S12E82 & 1990 & 250 & Yes & No \\
\hline SOL2014-03-29T17:48 & $\mathrm{X} 1.0$ & N11W32 & 2017 & 150 & Yes & No \\
\hline SOL2014-04-25T00:23 & $\mathrm{X} 1.3$ & S17W91 & 2035 & 70 & Yes & No \\
\hline SOL2014-06-10T11:42 & $\mathrm{X} 2.2$ & S15E80 & 2087 & 0 & Yes & No \\
\hline SOL2014-06-10T12:52 & $\mathrm{X} 1.5$ & S17E82 & 2087 & 0 & Yes & No \\
\hline SOL2014-06-11T09:06 & $\mathrm{X} 1.0$ & S18E65 & 2087 & 100 & Yes & No \\
\hline SOL2014-09-10T17:45 & $\mathrm{X} 1.6$ & N14E02 & 2158 & 420 & Yes & Yes \\
\hline SOL2014-10-19T05:03 & $\mathrm{X} 1.1$ & S13E42 & 2192 & 1240 & No & No \\
\hline SOL2014-10-22T14:28 & X1.6 & S14E13 & 2192 & 2410 & No & No \\
\hline SOL2014-10-24T21:46 & $\mathrm{X} 3.1$ & S16W21 & 2192 & 2740 & No & No \\
\hline SOL2014-10-25T17:08 & $\mathrm{X} 1.0$ & S16W31 & 2192 & 2510 & No & No \\
\hline SOL2014-10-26T10:56 & $\mathrm{X} 2.0$ & S18W40 & 2192 & 2570 & No & No \\
\hline SOL2014-10-27T14:47 & $\mathrm{X} 2.0$ & S17W52 & 2192 & 2750 & No & No \\
\hline SOL2014-11-07T17:26 & $\mathrm{X} 1.6$ & N15E33 & 2205 & 360 & Yes & No \\
\hline
\end{tabular}

${ }^{\text {a }}$ Spot area in millionths of the solar hemisphere $\left(3 \times 10^{12} \mathrm{~cm}^{2}\right)$, at 00:00 UT. 
region. The flare ribbons had large separations early in the process, suggesting reconnection high in the corona and remote from the photospheric inversion line. As with SOL2011-1113, the light curves give the appearance of a LDE (a "long-duration" or "long-decay" event; see e.g. Kahler, 1992). Thalmann et al. (2015) suggested that the non-thermal electron distribution was very steep during the entire flare, and that the total energy was relatively high for its GOES class. Sun et al. (2015) also studied this unusual region and found that although it was physically very large, its core had only weak non-potentiality, again suggesting that the over-lying field was relatively strong.

Another non-eruptive event (SOL2004-07-14) shows an extended period of contraction of over-lying loops in an extended $(\approx 30$-min) pre-impulsive phase (Kushwaha et al., 2015). The contraction coincided with plasma heating. This was followed by a normal impulsive phase during which the eruption of a flux rope occurred. The flux rope, however, remained confined beyond a certain height: such events are typically referred to as failed eruptions (e.g. Zirin et al., 1969; Ji et al., 2003; Gilbert, Alexander, and Liu, 2007; Joshi et al., 2015). In SOL2004-07-14, multiple coronal microwave sources are detected along the trajectory, possibly caused by the interaction of the contracting magnetic flux rope with the surrounding magnetic field ( $c f$. Fárník et al., 2003). Both the pre-flare situation and the over-lying magnetic field strength and orientation appear to play key roles in determining whether or not a full eruption can occur.

\section{Implications for CMEs from Other Stars}

One of our interests in studying X-flares is that they are the largest flares seen on the Sun and the closest in energy to stellar flares seen in G-type stars. The largest ever flare total bolometric energy released on the Sun is of the order of $10^{32}$ erg (Kretzschmar, 2011; Schrijver et al., 2012). However, flares on other solar-type stars have an energy which is 10 to $10^{4}$ times that of the largest solar flare (Schrijver and Beer, 2014). The Sun is observed continuously by a fleet of spacecraft and we can determine, after the event, whether there is a CME associated with the flare or not, and the potential impact on the Earth's environment. It is obviously not a trivial task to determine whether another solar-type star has produced a CME with a large flare, but understanding that is of great importance to understanding the likely environment around exoplanets. (Drake et al., 2013) made comparisons of solar power-law relationships to stellar energies to extrapolate what mass loss may be possible from solar-like stars. It is clear that there is potential for significant mass loss (see also Osten and Wolk, 2015).

Only some limited indirect evidence has been reported for stellar CMEs. One signature may be excessive absorption during X-ray flare events (e.g. Ottmann and Schmitt, 1996; Tsuboi et al., 1998), perhaps due to the presence of cool material from erupting filaments. Blue-shifted $\mathrm{H} \alpha$ emission during flares, indicating velocities of order $100 \mathrm{~km} \mathrm{~s}^{-1}$, also support the presence of CMEs (Montes et al., 1999; Fuhrmeister and Schmitt, 2004). Complex behavior of optical and ultraviolet lines during a large flare on the M dwarf AD Leo was interpreted in terms of various phases of a mass ejection including motion of filaments, chromospheric condensation, prominence oscillations, and the prominence eruption itself (Houdebine et al., 1993). The CME was suggested to be ejected at a velocity of $5800 \mathrm{~km} \mathrm{~s}^{-1}$, carrying a kinetic energy $\approx 300$ times larger than the total emitted UV energy, or $5 \times 10^{34} \mathrm{erg}$ (Houdebine, Foing, and Rodono, 1990).

The recent results from Kepler have allowed a larger statistical study of large flares on other solar-type stars. For example, Maehara et al. (2015) have studied 187 'super flares' 
and found a power-law distribution of the occurrence frequency and flare energy which is not too dissimilar to equivalent power-laws on the Sun (see e.g. Figure 3 in Schrijver et al., 2012). In addition they found a relationship between the flare duration and the flare energy. Some physical processes on solar-type stars may be similar to those on the Sun, with the additional complexity that many of the G-type stars will be faster rotators and the sunspot groups may be larger (Candelaresi et al., 2014). What certainly is not clear is under what situations a CME is produced on other stars.

In this paper we explore $42 \mathrm{X}$-class solar flares that have been observed since SDO was launched in 2010 until November 2014. Our aim was to determine whether there was any particular characteristic that could distinguish between large eruptive flares and non-eruptive flares, with the end goal of applying that knowledge to stellar flares.

\section{Data Analysis}

We have used multiple instrument data from multiple spacecraft in order to probe the $\mathrm{X}$ flares and seek answers to the questions we have posed. The data sources that we used are summarized here, and their implications for our questions are addressed in Section 5.

\subsection{GOES}

The solar flare classification we use throughout this paper is that derived from the X-ray spectrometers (XRS) on the GOES spacecraft (1-8 ̊ band). This defines flares logarithmically as $\mathrm{A}, \mathrm{B}, \mathrm{C}, \mathrm{M}$, and $\mathrm{X}$, where the peak fluxes are in the range $10^{-7}-10^{-2} \mathrm{~W} \mathrm{~m}^{-2}$. Each logarithmic X-ray class is divided into a linear scale from 1 to 9 (e.g., X3 means $3 \times 10^{-4} \mathrm{~W} \mathrm{~m}^{-2}$ ). The GOES energy itself represents only a small fraction, typically below $1 \%$, of the total flare energy (e.g. Emslie et al., 2012), and the GOES spectral response peaks at photon energies well above $k T$, where $T$ is the dominant flare temperature (e.g. White, Thomas, and Schwartz, 2005).

We have used the parent 2-s GOES data sampling to define time scales for each event: e-folding rise and decay times, and durations at half maximum (FWHM) of the $1-8 \AA$ band. These time scales are not the standard ones that NOAA reports, but have less dependence on the probably unrelated background flux level. This is also a measurement made on stellar light curves and is typically used in the optical band.

\subsection{The Hinode EUV Imaging Spectrometer (EIS)}

The Hinode/EIS (Culhane et al., 2007) is a scanning slit spectrometer observing in two wave bands in the EUV: $170-210 \AA$ and $250-290 \AA$. The spectral resolution is $0.0223 \AA$ pixel $^{-1}$, which allows velocity measurements of a few $\mathrm{km} \mathrm{s}^{-1}$. We analyzed data from EIS to explore the temperature at which the flows change from red-shifted to blue-shifted during a flare. The standard calibration was used through the routine eis_prep. Additionally, the slit tilt and the orbital variation of the line position were corrected. For each pixel we fitted the line using a single Gaussian profile producing an intensity and Doppler-velocity "sparse image" for each raster scan. 


\subsection{SDO/EVE}

The Extreme ultraviolet Variability Experiment (EVE; Woods et al., 2012) onboard SDO measures the solar EUV irradiance from 6 to $105 \mathrm{~nm}$ with spectral resolution $(0.1 \mathrm{~nm})$, at $10 \mathrm{~s}$ cadence available for most of the interval we study. These data and those from GOES/XRS represent simple Sun-as-a-star observations with no image resolution.

\subsection{Areas}

An active region typically consists of sunspots and their associated plage, both of which indicate the presence of intense magnetic fields. We make use of spot areas from NOAA and SDO here, noting that the spot/plage area fractions of an active region vary by a large factor during the evolution of a given region (e.g. LaBonte et al., 1984).

\subsection{SDO/AIA}

SDO/AIA observes the Sun in seven EUV and three UV channels with a pixel size of 0.6 arc$\mathrm{sec}$ and a time cadence of $12 \mathrm{~s}$. AIA data were analyzed to study the light curves of the flaring regions, and to study the temporal and spatial changes with temperature of the X-flares.

\subsection{Hard X-Rays and $\gamma$-Rays}

There are two sources of hard X-ray and $\gamma$-ray data during our interval of interest, the Reuven Ramaty High-Energy Solar Spectroscopic Imager (RHESSI; Lin et al., 2002) and the Fermi Gamma-ray Space Telescope (FERMI; Atwood et al., 2009), neither of which have complete time coverage because of orbit properties. FERMI is designed mainly to probe non-solar high-energy phenomena, whereas RHESSI is optimized for the Sun. While not a solar-dedicated facility, FERMI has detected many solar flares since its launch. It carries two instruments: the Large Area Telescope (LAT), which provides observations of gamma rays in the energy range $20 \mathrm{MeV}$ to $300 \mathrm{GeV}$, and the Gamma-ray Burst Monitor (GBM), which is sensitive to energies between $8 \mathrm{keV}$ and $40 \mathrm{MeV}$. We make use of the LAT data only to determine whether or not a flare had an associated high-energy gamma-ray signature.

\subsection{SOHO/LASCO}

The Large Angle Spectroscopic Coronagraph (LASCO; Brueckner et al., 1995) is a coronagraph package which carries two externally occulted coronagraphs that observed from 1.5 to 30 solar radii. We made use of the LASCO CME catalog ${ }^{1}$ in order to determine whether the X-flares had CMEs associated with them.

\section{The Flares}

We choose a time period from when SDO/AIA data were available in May 2010 until November 2014. During this period $42 \mathrm{X}$-flares occurred and nine had no CMEs of any significance. Table 1 summarizes the X-flares. The CMEs were determined from the LASCO

\footnotetext{
${ }^{1}$ http://cdaw.gsfc.nasa.gov/CME_list/index.html
} 
Figure 1 Flare magnitudes compared with the sunspot areas (in millionths of solar hemisphere, MSH) in the source regions. The blue points are the events without CMEs, and the red points are those with CMEs. All regions have longitudes within $\pm 75^{\circ}$ of central meridian; boxed points come from AR 2192.

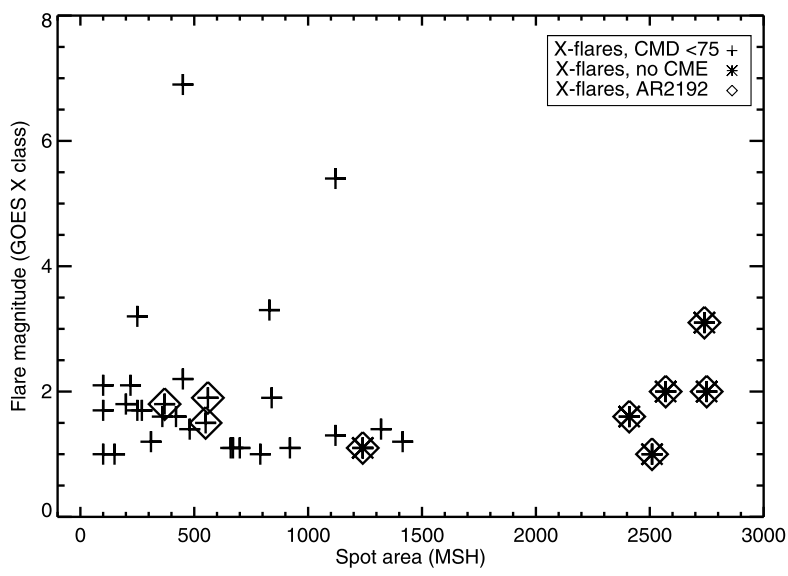

catalog. The events were thus described as either eruptive or non-eruptive. Note, however, that this is an extreme case of small-number statistics, since 6/9 of the non-eruptive flares in our sample came from the single region NOAA 12192. We also determined whether the events had solar energetic particles (SEPs) related to them or not. It is generally accepted that SEPs can be accelerated through two methods (Masson, Antiochos, and DeVore, 2013) - through impulsive flare that inject particles in the heliosphere via open field lines and through CMEs through which shock fronts play an important role in the acceleration process (Reames, 2013). There were many examples of there having been an eruption but no SEPs found. There were no examples where there was no eruption but SEPs were found.

As a simple overview of these events, we show the correlation between flare magnitude and sunspot area in Figure 1. Indeed there is no apparent correlation, in spite of the fact that our sample contains as many as 42 events. Thus we confirm that X-class flares can occur in regions with minimal sunspot area. The next subsections describe the questions we aim to address in order to challenge preconceptions of the behavior of X-flares and also to search for a signature distinguishing feature between flares with and without associated eruptions.

\subsection{Do All Eruptive Flares Have Long Durations?}

Some CME-associated flares have distinctly long decay times in soft X-rays, a fact reflected in the Pallavicini classification and described, for example, by Kahler (1992). On the other hand, some highly impulsive flares, with short decay times, also have CME associations. We revisit this question for our X-class flare sample here. The LDE association could reflect the influence of the large-scale field on eruptive flares. The CSHKP model envisions the gradual reconnection of higher and higher fields, with correspondingly longer time scales. The clear presence of loop arcades and soft X-ray cusp structures in many such events encourages this view (e.g. Svestka, 1986).

Several statistical studies have already tackled this question. Harrison (1995) explored this relationship in detail to examine the view that the brighter and longer-duration flares are more likely to be associated with CMEs. He found that CME onsets are associated with flares of any duration with $\approx 6 \%$ of flares having less than 1 hour duration and $60 \%$ under two hours. The peak GOES intensities of CME-associated flares could be of any value, but they are systematically larger than for the average solar flare. Andrews (2003) also carried out a statistical study of $311 \mathrm{M}$ and X-class flares and their SOHO/LASCO and EIT datasets. 
This study found that $40 \%$ of the M-class flares did not have an associated CME. This also supports the work of (Harrison, 1995) where the intensity and duration of flares were found not to guarantee a CME association. More recent statistical work also making use of the SOHO/LASCO data was carried out by (Yashiro et al., 2005) who analyzed $1301 \mathrm{X}$-ray flare events. These authors found that the CME-associated flares increased monotonically with flare GOES class from about $20 \%$ for C-class flares to $100 \%$ above the X3 level. Our sample (see Table 1) has extended this limit very slightly, noting the X3.1 event SOL201410-24. Yashiro et al. (2005) found that CMEs associated with the X-flares were faster and have wider extents, measured as the opening angles, than those associated with the weaker C-class flares.

In this section we explore the durations of X-flares for those with and without CMEs by analyzing the GOES X-ray data. The NOAA time scales have an ad hoc definition of the $1-8 \AA$ onset time, and take the return to $50 \%$ of the peak flux as the end time. These definitions have obvious systematic biases in terms of physical properties. Here we instead define the rise and fall times as logarithmic derivatives of the flux $S$, or $\tau=S / \dot{S}$, taken at the half-maximum times. These definitions also have systematic biases, in that they ignore the interesting complexity of the GOES light curves, but they definitely reduce the biases in the NOAA definitions. Our method takes the pre-flare minimum (also an ad hoc assumption) and computes the FWHM and e-folding rise and fall times based on the excess 1-8 $\AA$ flux above this background level. One remaining systematic bias in this approach is that many events show a two-stage exponential time decay; measuring the e-folding decay time at the half-maximum point may systematically miss the longer time scale, if present, but it does reflect the energetics of the flare (the impulsive phase) more closely.

Figure 2 shows the results of using the new time-scale definitions. The flare duration with flare date (top panel) shows a clearly non-random pattern, but there is no clear discrimination between events with and without CMEs. The lower figure shows the FWHM decay time $v s$. the FWHM rise time. In this case there is an approximate linear relationship between the rise and decay times, with a longer rise time leading to a longer decay time. However, there is again no distinguishing behavior for the flares with no associated CME. Figures 3 and 4

Figure 2 Top panel: flare duration (FWHM) vs. date of event. Crosses indicate eruptive event, diamonds non-eruptive. Bottom panel: decay time vs. rise time. These correlate, but they reveal no distinguishing difference between the two classes of events.
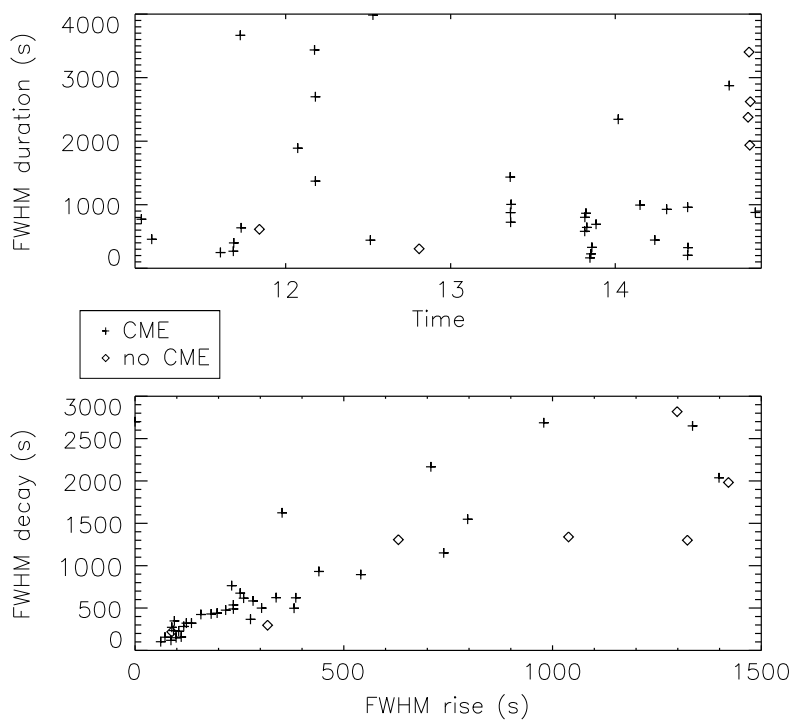

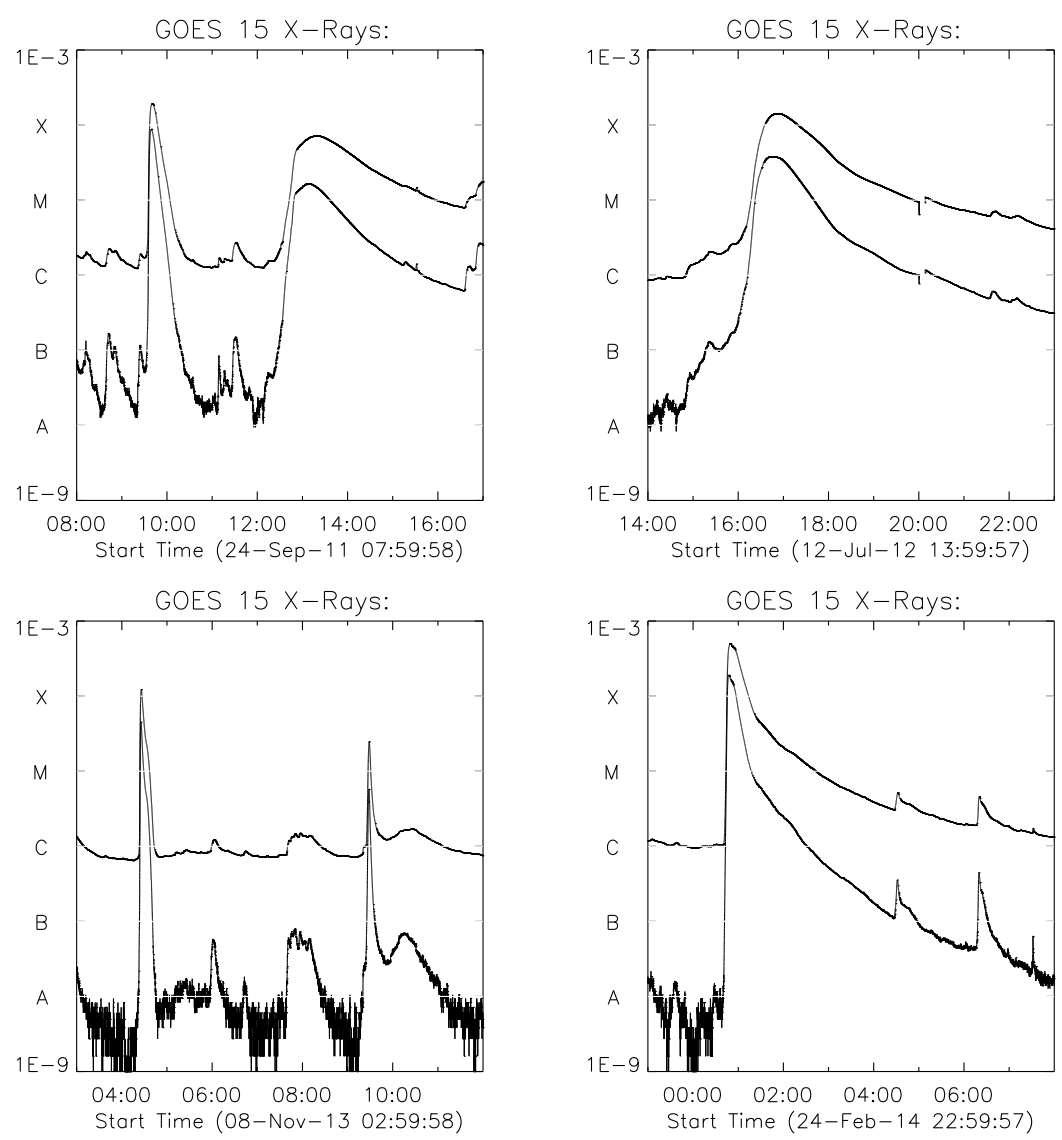

Figure 3 GOES soft X-ray light curves for four eruptive flares, each plot with the same $9 \mathrm{~h}$ time range.

show examples of GOES lightcurves for events that are eruptive and those that are not. It clear that in both cases the behavior can be either very impulsive and short-lived or longduration, but not always with an extended tail in both categories.

The lack of correlation between duration and eruptive behavior is maybe unexpected when the 'standard' flare model is considered. As discussed in the introduction, this may be due to the fact that the over-lying field is important in the process. The flaring process itself, in the core of the active region, will determine whether or not an eruption occurs. It is also clear that to understand each individual case, detailed modeling is required.

The statistical conclusions cannot be strong ones here, both because of the small sample but also because of the clear non-randomness of the occurrence of events: the 41 events come from only 21 unique active regions, with the unusual region NOAA 12192 producing six of the 41 events.

\subsection{Does Spot Area Correlate with Flare Magnitude?}

Figure 1 shows that no spot area-flare magnitude correlation exists within our database, yet it is widely believed that active regions with the most complex and largest sunspot groups are more likely to produce more powerful flares (Sammis, Tang, and Zirin, 2000). Such 

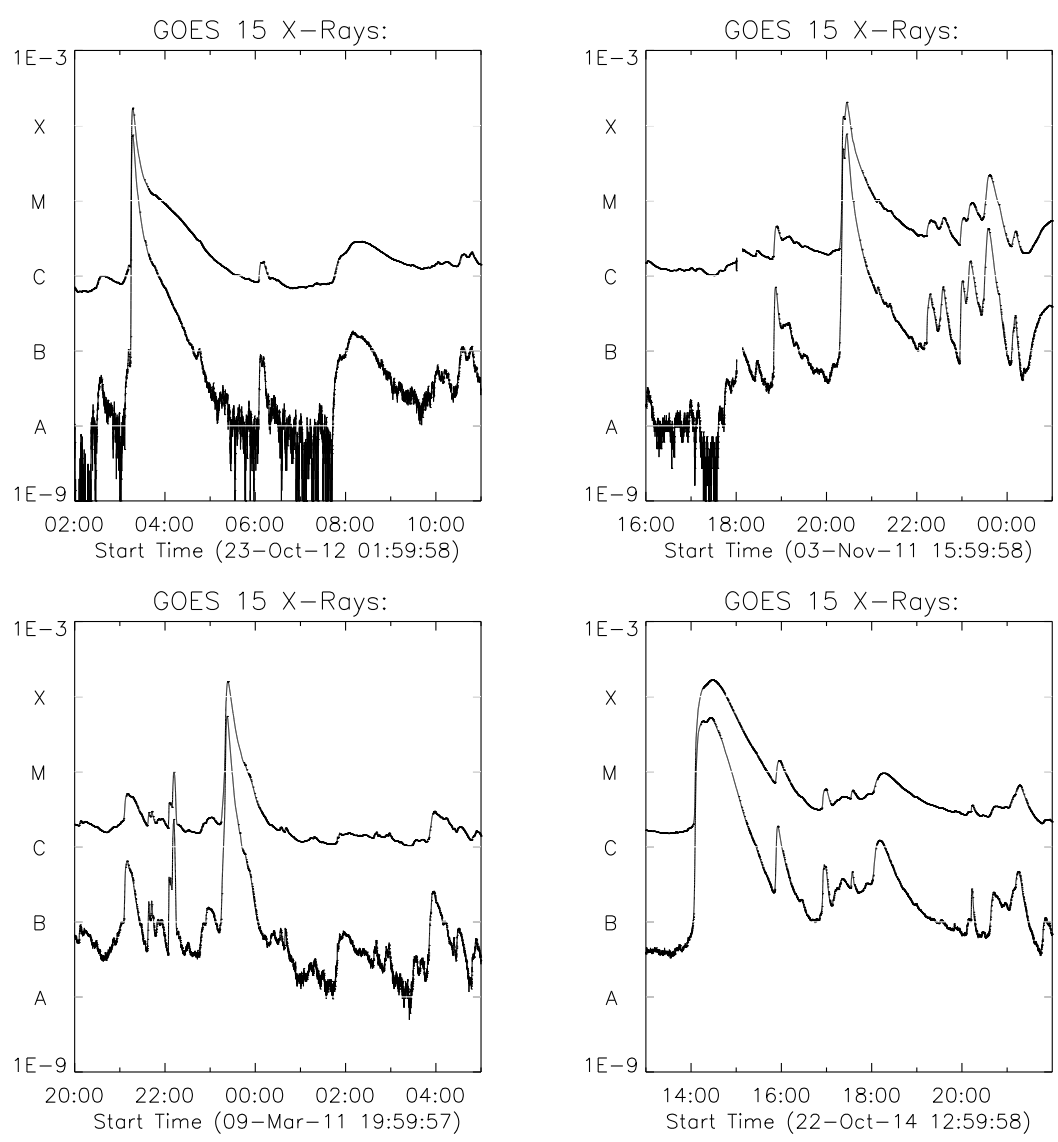

Figure 4 GOES soft X-ray light curves for four non-eruptive flares, with the same time scale as that of Figure 3.

characteristics are measured in flares and used as input into space weather flare forecasts, and so it is important to understand clearly what is meant - our result seems to contradict the conclusions of Sammis, Tang, and Zirin (2000). The explanation probably lies in the fact that Sammis, Tang, and Zirin (2000) used the maximal flare and maximal area for a given region, ignoring the time variation; their resulting correlation presumably relates more to the availability of a magnetic field than to its organization into sunspots per se.

\subsection{Does a Larger Spot Area Mean a Longer Duration Flare?}

As discussed in the previous subsections, the duration of the flare is important in terms of the amount of energy emitted in the X-ray and EUV. Here, we measure the size of the active regions in which the $\mathrm{X}$-flares occurred using HMI continuum data. Only active regions that were within $45^{\circ}$ of disk center were analyzed. After subtracting the center-to-limb variation (so-called limb darkening) and the projection effect, the area was assumed to be that with intensity less than $0.85\left\langle I_{\mathrm{QS}}\right\rangle$, where $\left\langle I_{\mathrm{QS}}\right\rangle$ is the averaged intensity of the quiet Sun. This is equivalent to the total area of umbrae and penumbrae. Figure 5 shows the results. There is a general relationship between the duration of the flare and the area of the sunspot group 
Figure 5 Flare duration $v s$. sunspot area, weakly suggesting a correlation. The pluses show active regions that had eruptive flares and the diamonds, mostly from AR 2192, non-eruptive flares.

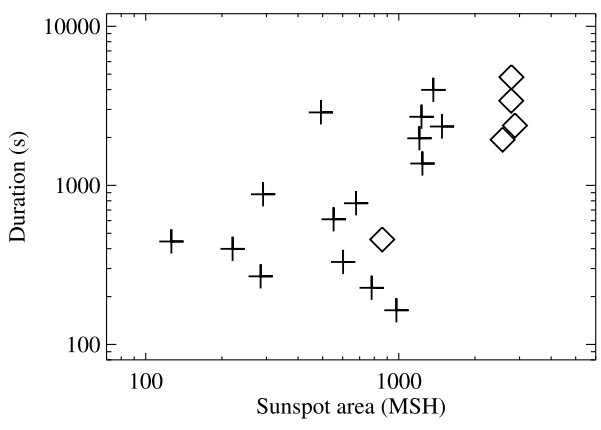

with scatter. Although only two active regions for events that are non-eruptive are shown (ARs 11166 and 12192), most of them are seen toward the right-hand side of the plot. This may indicate that if the active region is larger, it may have stronger over-lying magnetic fields, which could inhibit the ascending filament from becoming a CME (Sun et al., 2015).

\subsection{What Fraction of an Active Region is Involved in a Flare?}

Following on from the previous section, the area of the active region actually involved in the flare is explored. This is determined by analyzing the size of the flare ribbons. Previous studies of flaring active regions have shown that only a fraction of their field is involved in the conversion of energy. This can be estimated by measuring the area swept by the flare ribbons, since they delimit the area where the magnetic flux has reconnected. For example, Qiu et al. (2007) and Kazachenko et al. (2012) reported on a dozen of flaring events and found that the percentage of an active region involved in a flare ranged between $10 \%$ and $30 \%$, on average. How does this proportion compare with our set of X-class flares?

The active region itself is defined from the HMI magnetograms where $B_{z}>100$ gauss (G). Several other thresholds have been chosen for comparison, but this value is chosen for consistency between the different X-flare regions. It is also the threshold of background transverse field error or noise estimated in Wiegelmann and Inhester (2010). The HMI magnetograms are used. In these cases only the disk active regions were analyzed to avoid issues with line-of-sight effects. Figure 6 shows an example of how the flaring region was defined. Table 2 summarizes the results. There is a range from $7 \%$ to $41 \%$ flaring region as a percentage of the total active region. It might be expected that the larger the area delimited by the flare ribbons, the higher altitude reconnection will take place (from the standard flare model scenario). If this is the case, then it might be expected that the higher altitude reconnection takes place, the more chance there is for plasma to find a means to escape from the Sun and form a CME. However, there is no significant difference between the eruptive and non-eruptive case. This may support the idea that the behavior of flare ribbons also depends on the flux surface they interact with: in some cases the flare ribbons of the leading, compact polarity do not move much, while the ones in the trailing polarity do. An example of extended flare ribbons right at the start of the flare was described by Thalmann et al. (2015).

Studying the ratio of the surface that is typically involved in the flare for an active region can also help estimate the energy associated to stellar flares. As was discussed in Aulanier et al. (2013), the size of an active region as well as the magnetic field intensity concentrated in a main bipole can help obtain an upper limit on solar as well as stellar flares. Such estimations may be tested in the future with more accurate estimations of active region on stars (or starspots) as well as the magnetic field intensity obtained with spectropolarimetric observations. 

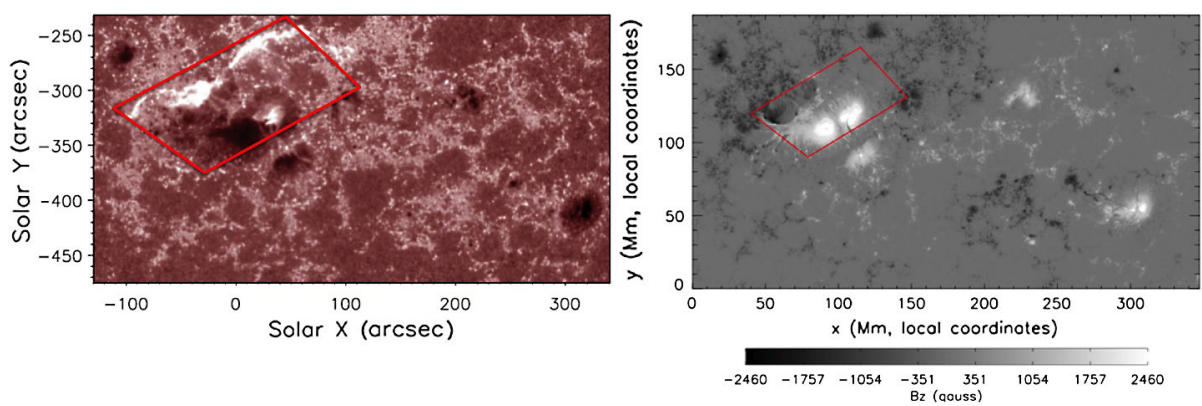

Figure 6 Active region NOAA 1520. On the left shows the flare ribbons as seen in the $1700 \AA$ bandpass, and the right shows the magnetogram. The magnetogram is shown in the solar local coordinates. The red box highlights the area that the flare ribbons encompass. This was an eruptive X-class flare.

Table 2 A table summarizing the date, location, GOES class, whether or not there was a CME, and the ratio of the region of the active region involved in flaring defined by the flare ribbons relative to the total area of the active region.

\begin{tabular}{llllc}
\hline Date & Location & GOES class & CME? & Area fraction (\%) \\
\hline SOL2011-02-15T01:56 & S20W10 & X2.2 & yes & 12 \\
SOL2011-03-09T01:56 & N08W11 & X1.5 & no & 16 \\
SOL2011-08-09T08:05 & N14W69 & X6.9 & yes & 9.3 \\
SOL2011-09-06T22:20 & N14W18 & X2.1 & yes & 4 \\
SOL2011-09-07T22:38 & N14W31 & X1.8 & yes & 41 \\
SOL2012-03-07T00:24 & N18E31 & X5.4 & yes & 36 \\
SOL2012-03-07T01:14 & N15E26 & X1.3 & yes & 13 \\
SOL2012-07-12T16:49 & S13W03 & X1.4 & yes & 13 \\
SOL2013-11-08T04:26 & S14E15 & X1.1 & yes & 7 \\
SOL2013-11-10T05:14 & S14W13 & X1.1 & yes & 7 \\
SOL2014-10-22T14:28 & S14E13 & X1.6 & no & 12.7 \\
SOL2014-10-24T14:28 & S16W21 & X3.1 & no & 15.5 \\
\hline
\end{tabular}

\subsection{Do X-Flares with No Eruptions Have More Non-thermal Energy?}

The energy conversion that occurs in a flare has, in a very simplistic way, three routes to be expended (e.g. Canfield et al., 1980; Wu et al., 1986; Emslie et al., 2012). The first is non-thermal energy, observed through hard X-ray and gamma-ray emission, the second is thermal emission, observed through heating of the plasma, and the last is via the bulk motion observed if there is an ejection or CME. A high percentage of the non-thermal energy will be converted into thermal energy at a later stage. Some of this can be observed through the evaporation process, again to be radiated at a later stage. This will be discussed in the next section. In this section we study whether the non-eruptive flares have more non-thermal energy relative to eruptive flares of a similar GOES class. To investigate this premise we fitted the FERMI data over the range $15-150 \mathrm{keV}$ using a combination of a thermal and a thick-target model, and from this we derived the associated non-thermal energy. Only 22 of the $42 \mathrm{X}$-flares were observed by FERMI, and Figure 7 illustrates the non-thermal energy derived from the spectral fitting as a function of time and also with GOES intensity. As 
Figure 7 The top plot shows the flare non-thermal energy versus time, with the eruptive and non-eruptive flare identified. The lower plot shows the non-thermal energy versus the GOES intensity.

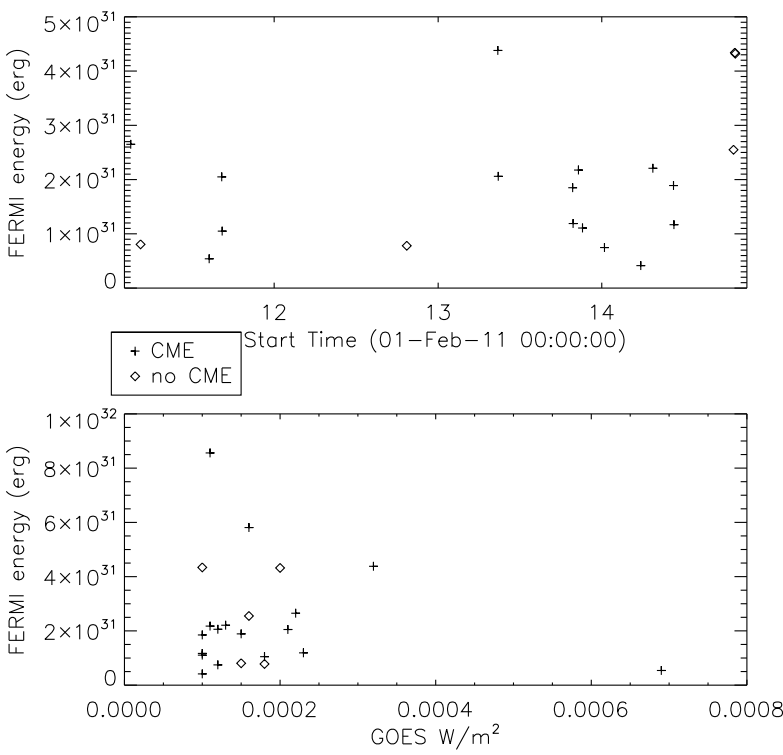

was pointed by Thalmann et al. (2015) the October 2014 X-flares have some of the highest energies measured. However, the other regions that have no eruptions showed lower nonthermal energies and regions that have eruptions also reach similar non-thermal energies than those with no eruptions. Once again, there appears to be no clear distinction between the eruptive and non-eruptive flares.

\subsection{Evaporation in Flares - Is This Different in Eruptive and Non-eruptive Flares?}

As discussed in the introduction, evaporation of chromospheric plasma is expected following the impact of a non-thermal energy beam in the chromosphere. In the work by Milligan and Dennis (2009) was found evidence of evaporated plasma in emission lines with temperatures from $2-16 \mathrm{MK}$. The up-flow velocity was found to scale with temperature. The lower temperature lines from $0.5-1.5 \mathrm{MK}$ were found to be red-shifted (down-flows) and were interpreted as being characteristic of explosive evaporation. These down-flows were found to occur at temperatures higher than expected before, which needs to be understood. In a study by Doschek, Warren, and Young (2013) evaporation of the hotter emission was found in multiple locations. Interestingly, observations were by chance made at the site close to the site of particle impact onto the chromosphere and the hotter emission had a source with no blue-shifted components, but with strong down-flows seen in cooler lines, such as Fe XIII and Fe XIV. It was hypothesized that this was due to a downward shock produced during the reconnection process. Recent results from the IRIS mission have also shown similar results but with strong down-flows seen in Fe XXI spatially coincident with the loop top - consistent with hot-retracting loops for example (Tian et al., 2014). Sadykov et al. (2015) found evaporation flows in the Fe XXI ion in a flare. Polito et al. (2015) found that after this blue-shifted plasma was observed, the Fe XXI intensity slowly moved from the footprints to the top of the flare loops. The location of the flows during a flare and the temperature they appear at are clearly important in order to try to understand the physical processes going on. Following on from the previous section, we studied the temperature at which the flows change from 

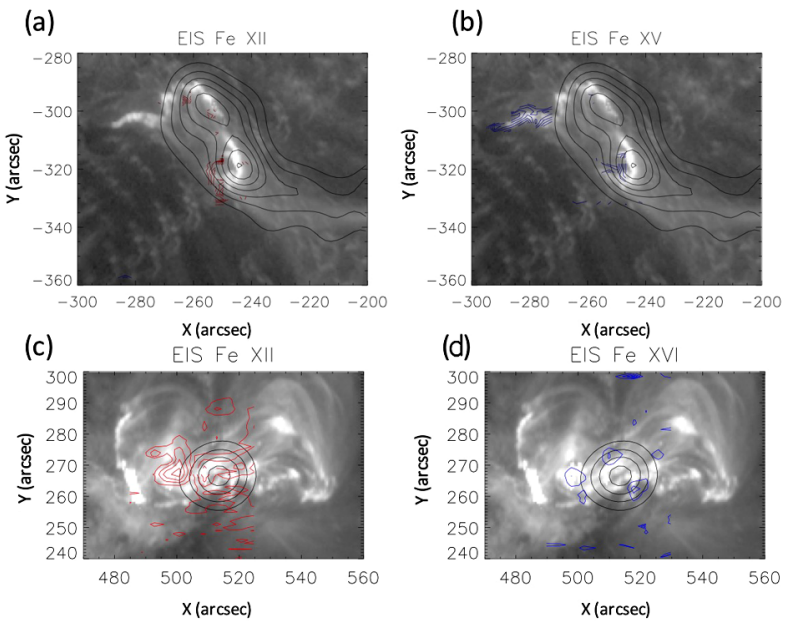

Figure 8 An example of the temperature behavior of the Doppler flows for an X-flare which is not-eruptive is shown in (a) and (b). The black and white image is a 193A image. The black contours are the $12-25 \mathrm{keV}$ RHESSI maps. (a) shows red contours which are Doppler velocity for the EIS Fe XII ion. (b) shows blue contours which are Doppler velocities for the EIS Fe XV ion. The red contours indicate red-shifted plasma and the blue indicate blue-shifted plasma. The velocity ranges between $20-60 \mathrm{~km} \mathrm{~s}^{-1}$. (c) and (d) show the same plots for an eruptive flare. The same behavior is seen in the red- and blue-shifted plasma.

red-shift to blue-shift in each of the X-flares that had EIS observations. The number of flares is limited due to the small field of view of the spectrometers, but we have examples of flares that are both eruptive and non-eruptive. We tested whether in non-eruptive flares the flows may appear at different temperatures due to different partition of energy. There were three examples for eruptive flares and five for non-eruptive flares. Figure 8 shows an example of a non-eruptive flare and of an eruptive flare. As in the paper by Milligan and Dennis (2009) the change between red- and blue-shifted plasma occurs above the temperature of the Fe $\mathrm{XII}$ ion. There is no difference between the eruptive and non-eruptive examples; hence there seems to be no difference in the location of the energy deposition.

\subsection{Is EUV Dimming Only Seen in Eruptive Flares?}

Coronal dimming, suggestive of a CME occurrence, first appeared in day-on-day changes in the white-light corona (Hansen et al., 1974) as "depletions". The Skylab observations revealed sudden changes in the X-ray emission corona as well - "transient coronal holes" (Rust and Hildner, 1976) - well before CMEs had been identified as such. The Yohkoh soft $\mathrm{X}$-ray data, with good sampling, revealed that these dimmings of the X-ray corona occurred systematically and could have a relationship with CMEs (Hudson and Webb, 1997). Sterling and Hudson (1997) found that the sources of the dimming were predominantly at the ends of a pre-flare sigmoid structure, suggesting that the dimming was caused by an erupting flux rope.

Thompson et al. (2000) imaged seven events observed in the EUV and found that dimmings and CMEs coincided in each case. These dimmings can be large scale, reaching large distances from the active-region core. Using SOHO data, Reinard and Biesecker (2008) carried out a statistical study to determine the link between EUV dimmings and CMEs. They found that indeed all such dimmings were associated with CMEs. Conversely not all CMEs 
were associated with dimmings; these uncorrelated events tended to be related to the slower CMEs. Spectroscopic observations using SOHO/CDS (Harra and Sterling, 2001) and more recently Hinode/EIS (Harra et al., 2007), have confirmed that the dimmings are regions of up-flowing expanding plasma, rather than temperature shifts ( $c f$. Hudson, Acton, and Freeland, 1996). Mason et al. (2014) described SDO/EVE data, which show the major dimmings clearly in integrated EUV Sun-as-a-star spectroscopy. These data, with $0.1 \mathrm{~nm}$ spectroscopic resolution and excellent signal-to-noise ratio, are available for our entire sample. The Mason et al. (2014) analysis used an automated technique to search for dimmings, and it found 263, again concluding that the major explanation of an EUV dimming was an actual ejection of a part of the pre-flare corona, consistent with the broad-band observations in white light and soft X-rays.

We illustrate the EVE dimming measurements in Figure 9, which displays light curves of SOL2012-03-07 in a range of ionization states of iron, Fe IX through Fe XXIV. Dimming appears in low-excitation states beginning near the peak of the flare in high-excitation states. These time series show all aspects of the flare/CME development insofar as they can be recognized in non-imaging observations. The lines at lowest temperatures, Fe XVIII-Fe XII, have the cleanest dimming signatures, implying that most of the CME material comes from the ambient corona rather than the active region itself.

Figure 9 provides an overview of some of the EVE spectroscopy, but may have too much information. Thus we plot more limited sets of data for SOL2012-03-07 and for the noneruptive event SOL2011-11-03 in Figures 10 and 11, respectively. These select four lines (Fe X, XV, XXI, XXIV) at peak (logarithmic) formation temperatures of [6.0, 6.4, 7.0, 7.3], respectively. The impulsive X-class flare SOL2011-11-03 did not involve a CME or a dimming, but within a few hours a remote event on the invisible hemisphere (Gómez-Herrero et al., 2015) produced a CME, SEPs, and apparently also the gradual dimming that one sees in Fe X here (Figure 11) beginning at about 4 November 00:30 UT.

\section{Discussion}

The aim of the paper was to explore whether there are differences in large flares between those that are eruptive and those that are not eruptive. We studied seven questions that either come from preconceptions about large flares, or a physical expectation of how energy will be distributed in $\mathrm{X}$-flares with or without CMEs. These included:

- Do large sunspot areas correspond to more powerful flares? In our sample, they do not, but averaged over an active-region lifetime, Sammis, Tang, and Zirin (2000) found a reasonable correlation. This behavior may suggest that the sunspots themselves have little to do with the flares, while of course the magnetic flux does.

- Do all eruptive flares have a long duration? It appears they do not. Some X-class flares with CMEs showed a very impulsive rise time, and short durations, and some X-class flares without CMEs were long-duration events.

- Do CME-related flares stand out in terms of active-region size vs. flare duration? This might be expected in the sense that if you have more wide-spread magnetic fields, then there is a greater volume in which reconnection can take place. There is indeed a rough correlation here. Moreover, we found the tendency that for the flare events with the same durations, CME-poor ARs have larger spot areas. This may be because larger ARs have over-lying fields to inhibit the CME eruptions. 


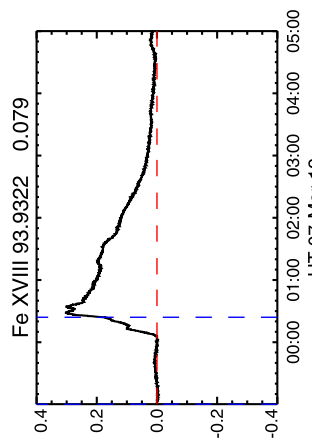

(z-w Mw) әоие!peג1

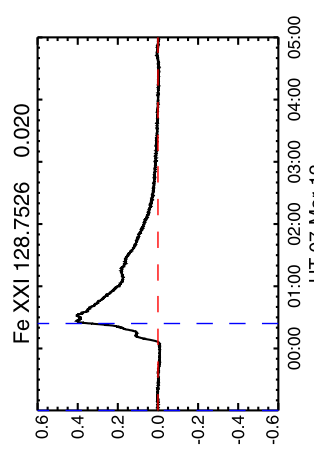

(z-u Mu) әэuе!peג1

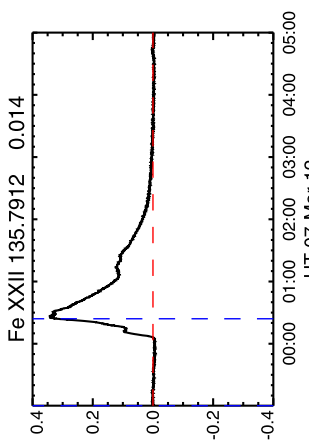

(ट-u Mu) әоие!peג1

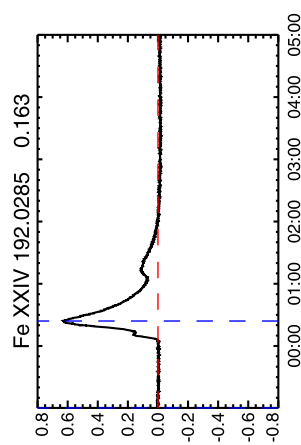

(z-u Mu) әэие!peג1

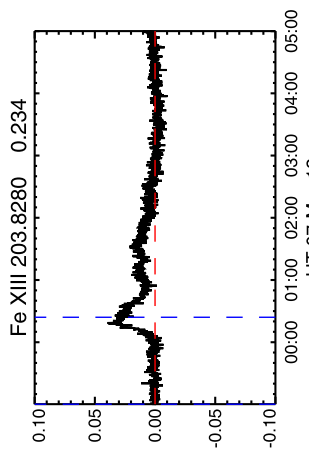

(z-u Mu) әэuе!peג1|

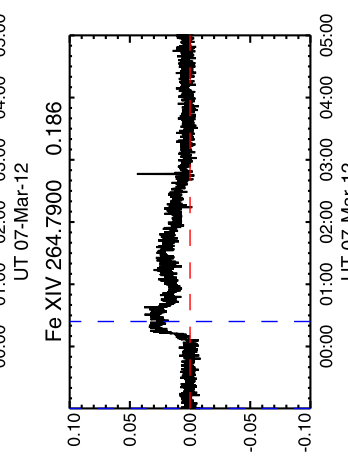

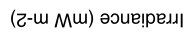

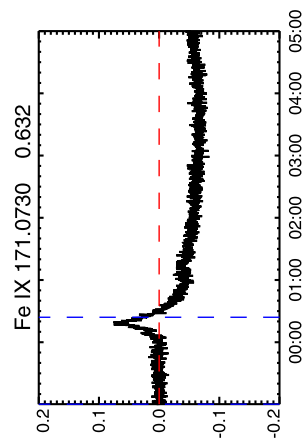

(乙-u Mu) әоие!peגᄉ|

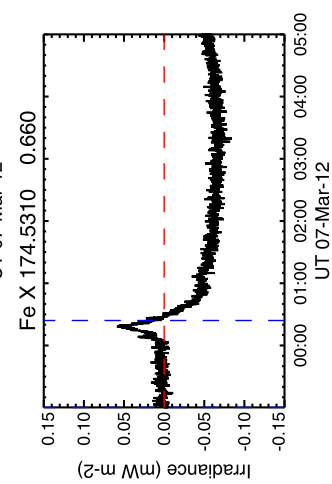

(z-w Mu) әоนе!peג|

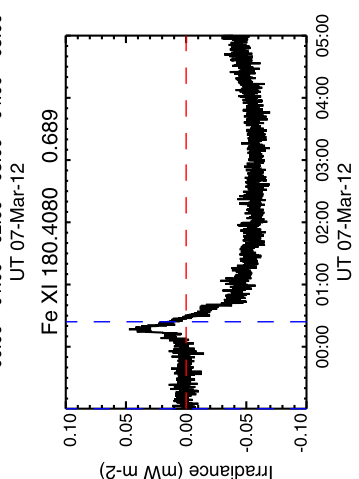

(z-u Mu) әэuе!peגа

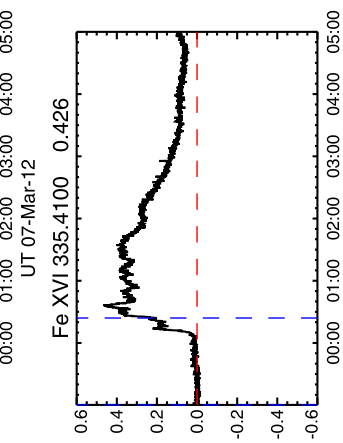

(z-u Mu) әэuе!peג1

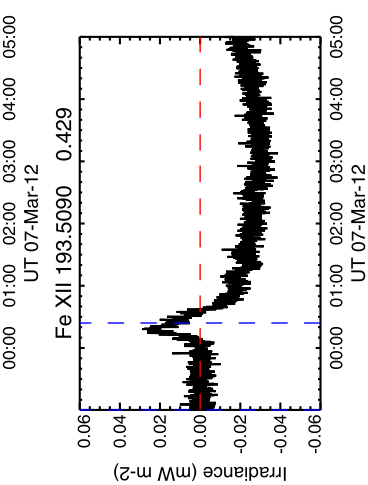

ํㅡㄹ

苞

$\Xi . \Xi$

$\geq \overline{0}$

$\times$ ․

is

छิ

$\forall 0$

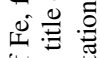

t)

证.

के

.

임

일

을

跑

$\exists$.

근

ฮ छ

क

을

용

光

एक

สิ 余

등

记 产

․․ㅇ

के 흔

o

돌

त

荡穿

。

की प्ञิ

蛋

元

范 을

ธ。 의

它要

원

금

तิ न्ञ

을

호

䡒

进

0.0

항

띠응

$>\frac{1}{1}$

핑혀

․ํ

क $₫$

a 3

농

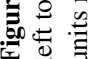



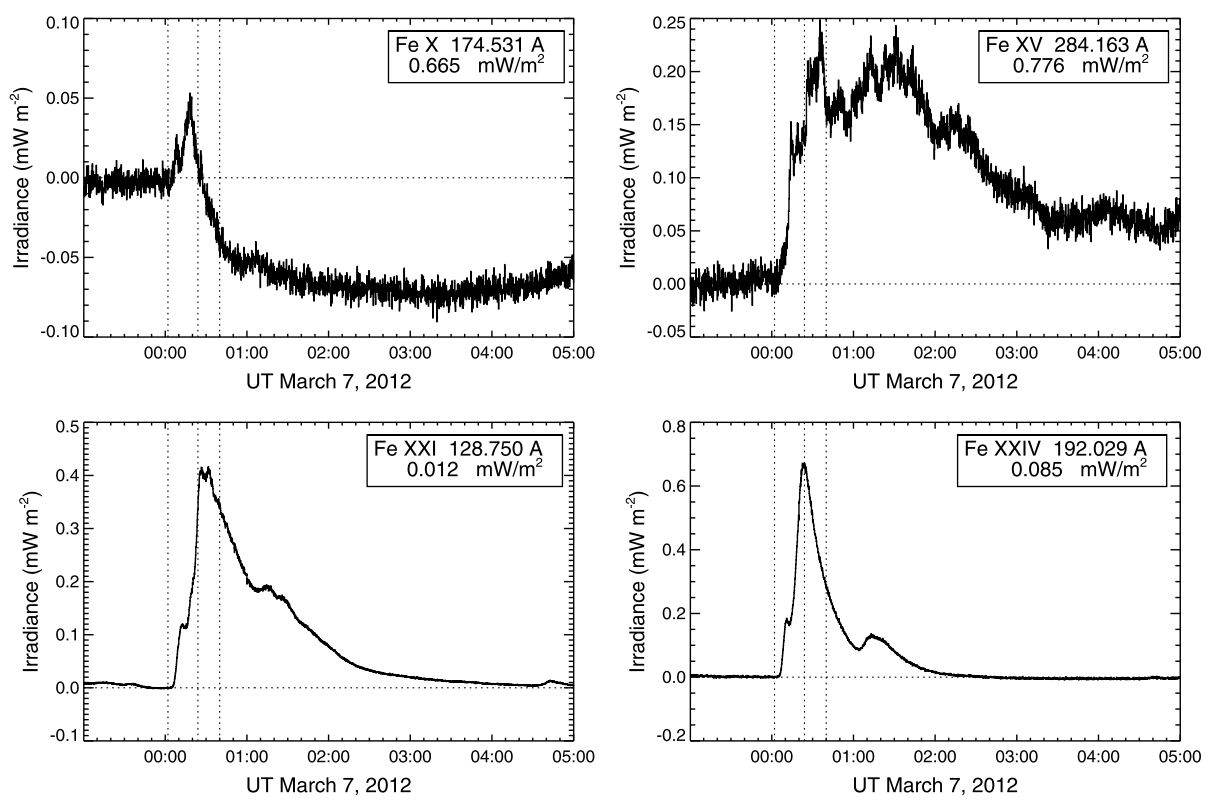

Figure 10 SDO/EVE light curves for SOL2012-03-07, in a similar format as before to that of Figure 9, but now with fewer lines and a shorter time interval; also an ion with the peak dimming signature (Fe $\mathrm{X})$ is in the upper left, with the hottest line (Fe XXIV) in the lower right. This event shows prompt dimming and had an accompanying CME.

- Is the occurrence of CMEs related to the fraction of active-region area involved? The hypothesis here is that larger flare ribbons would indicate higher altitude reconnection, which might make it easier for eruptions to escape. However, we found no difference between those with and without CMEs.

- Do X-flares with no eruptions have greater non-thermal energy? For those flares where non-thermal energy was estimated from the FERMI data there was no systematic difference between the flares with and without CMEs.

- Is the temperature dependence of evaporation different in eruptive and non-eruptive flares? Once again, we found no difference between flares with or without CMEs.

- Is EUV dimming only seen in eruptive flares? Yes - EUV dimming is seen for all eruptive $\mathrm{X}$-flares and the dimming will start within an hour after the peak of the flare.

The only characteristic that seems to show a consistent difference between flares with and without CMEs is that of coronal dimming. This can be observed distinctly in the spatially unresolved EUV light curves from EVE, in principle providing an ideal candidate for comparison with the stellar case. However, it is difficult to have similar spectral coverage for the stellar observations. The NASA Extreme Ultraviolet Explorer (EUVE; Bowyer, Malina, and Marshall, 1988), which stopped observing in 2001, had the closest overlap in wavebands covering 90-150 ̊, $180-350 \AA$. To our knowledge EUVE data provided no evidence for coronal dimming events. Modeling and theory should be developed to understand the dimming process not only for the Sun, but also for the case of larger sunspot groups, and on faster-rotating stars. It would be of use to simulate the optimum waveband where dimming could be seen, and to plan to cover this range in future stellar missions. In the solar case it is around $1 \mathrm{MK}$, but it may well be hotter in other stars (if the phenomenon actually ex- 

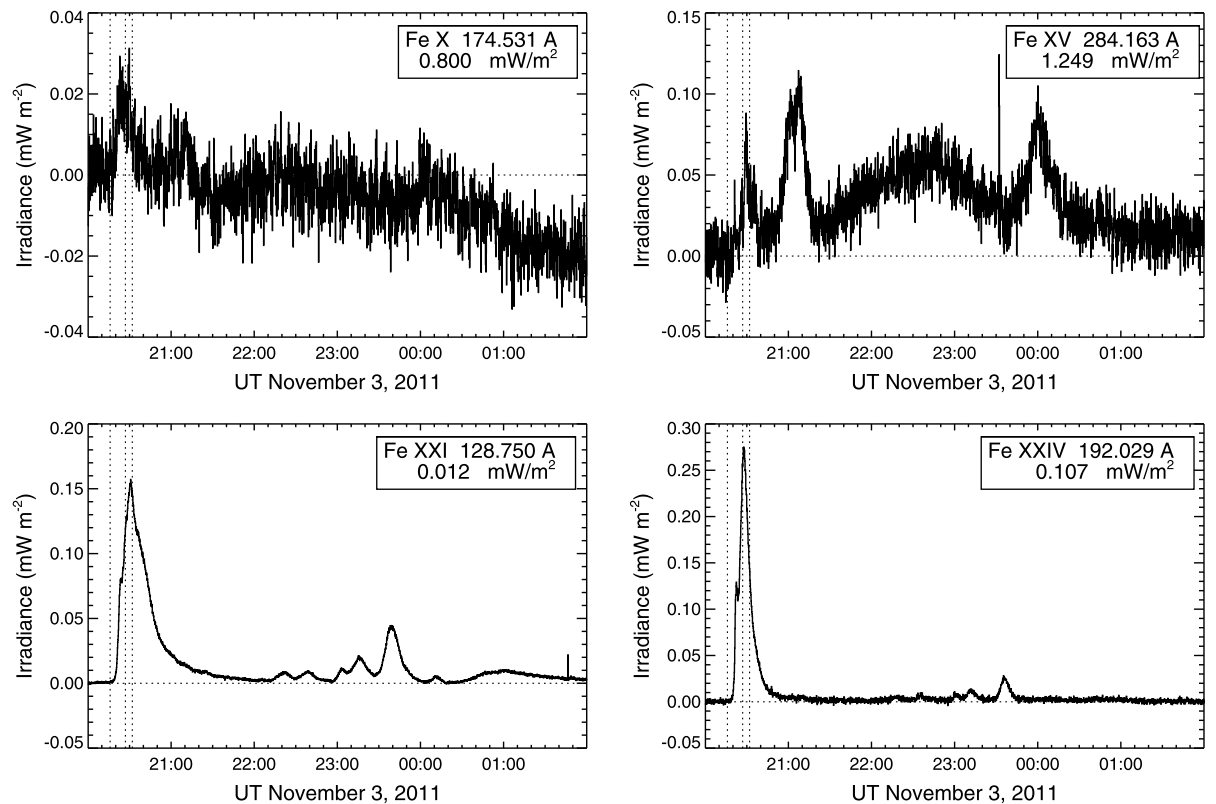

Figure 11 SDO/EVE light curves for SOL2011-11-03, in the same format as that of Figure 10. This event shows no prompt dimming and had no CME.

ists) with hotter quiescent coronae. We would expect this from more extensive and stronger magnetic fields on more active stars.

Acknowledgements The authors thank ISSI for the support of the solar-stellar team. This CME catalog is generated and maintained at the CDAW Data Center by NASA and The Catholic University of America in cooperation with the Naval Research Laboratory. SOHO is a project of international cooperation between ESA and NASA. The Solar Dynamics Observatory is a mission in the NASA Living With a Star program. Hinode is a Japanese mission developed and launched by ISAS/JAXA, with NAOJ as domestic partner and NASA and STFC (UK) as international partners. It is operated by these agencies in cooperation with ESA and NSC (Norway). TL acknowledges funding of the Austrian FFG within ASAP11 and the FWF NFN project S116601-N16. MJ thanks V. Bommier for providing the UNNOFIT-inverted maps used in the active-region area calculations.

Open Access This article is distributed under the terms of the Creative Commons Attribution 4.0 International License (http://creativecommons.org/licenses/by/4.0/), which permits unrestricted use, distribution, and reproduction in any medium, provided you give appropriate credit to the original author(s) and the source, provide a link to the Creative Commons license, and indicate if changes were made.

\section{References}

Andrews, M.D.: 2003, A search for CMEs associated with big flares. Solar Phys. 218, 261. DOI. ADS.

Atwood, W.B., Abdo, A.A., Ackermann, M., Althouse, W., Anderson, B., Axelsson, M., et al.: 2009, The large area telescope on the Fermi gamma-ray space telescope mission. Astrophys. J. 697, 1071. DOI. ADS.

Aulanier, G., Démoulin, P., Schrijver, C.J., Janvier, M., Pariat, E., Schmieder, B.: 2013, The standard flare model in three dimensions, II: Upper limit on solar flare energy. Astron. Astrophys. 549, A66. DOI. ADS.

Bowyer, S., Malina, R.F., Marshall, H.L.: 1988, The extreme ultraviolet explorer mission - Instrumentation and science goals. J. Br. Interplanet. Soc. 41, 357. ADS. 
Brueckner, G.E., Howard, R.A., Koomen, M.J., Korendyke, C.M., Michels, D.J., Moses, J.D., Socker, D.G., Dere, K.P., Lamy, P.L., Llebaria, A., Bout, M.V., Schwenn, R., Simnett, G.M., Bedford, D.K., Eyles, C.J.: 1995, The Large Angle Spectroscopic Coronagraph (LASCO). Solar Phys. 162, 357. DOI. ADS.

Candelaresi, S., Hillier, A., Maehara, H., Brandenburg, A., Shibata, K.: 2014, Superflare occurrence and energies on G-, K-, and M-type dwarfs. Astrophys. J. 792, 67. DOI. ADS.

Canfield, R.C., Cheng, C.-C., Dere, K.P., Dulk, G.A., McLean, D.J., Schmahl, E.J., Robinson, R.D., Jr., Schoolman, S.A.: 1980, Radiative energy output of the 5 September 1973 flare. In: Sturrock, P.A. (ed.) Solar Flares: A Monograph from Skylab Workshop II, Colorado Associated Univ. Press, Boulder, 451. ADS.

Carmichael, H.: 1964, A process for flares. In: Hess, W.N. (ed.) AAS-NASA Symposium on the Physics of Solar Flares, NASA SP-50, 451. ADS.

Culhane, J.L., Harra, L.K., James, A.M., Al-Janabi, K., Bradley, L.J., Chaudry, R.A., Rees, K., Tandy, J.A., Thomas, P., Whillock, M.C.R., Winter, B., Doschek, G.A., Korendyke, C.M., Brown, C.M., Myers, S., Mariska, J., Seely, J., Lang, J., Kent, B.J., Shaughnessy, B.M., Young, P.R., Simnett, G.M., Castelli, C.M., Mahmoud, S., Mapson-Menard, H., Probyn, B.J., Thomas, R.J., Davila, J., Dere, K., Windt, D., Shea, J., Hagood, R., Moye, R., Hara, H., Watanabe, T., Matsuzaki, K., Kosugi, T., Hansteen, V., Wikstol, Ø.: 2007, The EUV imaging spectrometer for Hinode. Solar Phys. 243, 19. DOI. ADS.

Doschek, G.A., Warren, H.P., Young, P.R.: 2013, Chromospheric evaporation in an M1.8 flare observed by the extreme-ultraviolet imaging spectrometer on Hinode. Astrophys. J. 767, 55. DOI. ADS.

Drake, J.J., Cohen, O., Yashiro, S., Gopalswamy, N.: 2013, Implications of mass and energy loss due to coronal mass ejections on magnetically active stars. Astrophys. J. 764, 170. DOI. ADS.

Emslie, A.G., Dennis, B.R., Shih, A.Y., Chamberlin, P.C., Mewaldt, R.A., Moore, C.S., Share, G.H., Vourlidas, A., Welsch, B.T.: 2012, Global energetics of thirty-eight large solar eruptive events. Astrophys. J. 759, 71. DOI. ADS.

Fárník, F., Hudson, H.S., Karlický, M., Kosugi, T.: 2003, X-ray and radio observations of the activation stages of an X-class solar flare. Astron. Astrophys. 399, 1159. DOI. ADS.

Fletcher, L., Dennis, B.R., Hudson, H.S., Krucker, S., Phillips, K., Veronig, A., Battaglia, M., Bone, L., Caspi, A., Chen, Q., Gallagher, P., Grigis, P.T., Ji, H., Liu, W., Milligan, R.O., Temmer, M.: 2011, An observational overview of solar flares. Space Sci. Rev. 159, 19. DOI. ADS.

Forbes, T.G., Acton, L.W.: 1996, Reconnection and field line shrinkage in solar llares. Astrophys. J. 459, 330. DOI. ADS.

Fuhrmeister, B., Schmitt, J.H.M.M.: 2004, Detection and high-resolution spectroscopy of a huge flare on the old M 9 dwarf DENIS 104814.7-395606.1. Astron. Astrophys. 420, 1079. DOI. ADS.

Gilbert, H.R., Alexander, D., Liu, R.: 2007, Filament kinking and its implications for eruption and reformation. Solar Phys. 245, 287. DOI. ADS.

Gómez-Herrero, R., Dresing, N., Klassen, A., Heber, B., Lario, D., Agueda, N., Malandraki, O.E., Blanco, J.J., Rodríguez-Pacheco, J., Banjac, S.: 2015, Circumsolar energetic particle distribution on 2011 November 3. Astrophys. J. 799, 55. DOI. ADS.

Gosling, J.T., McComas, D.J., Phillips, J.L., Bame, S.J.: 1991, Geomagnetic activity associated with earth passage of interplanetary shock disturbances and coronal mass ejections. J. Geophys. Res. 96, 7831. DOI.

Hansen, R.T., Garcia, C.J., Hansen, S.F., Yasukawa, E.: 1974, Abrupt depletions of the inner corona. Publ. Astron. Soc. Japan 86, 500. DOI. ADS.

Harra, L.K., Sterling, A.C.: 2001, Material outflows from coronal intensity "dimming regions" during coronal mass ejection onset. Astrophys. J. Lett. 561, L215. DOI. ADS.

Harra, L.K., Hara, H., Imada, S., Young, P.R., Williams, D.R., Sterling, A.C., Korendyke, C., Attrill, G.D.R.: 2007, Coronal dimming observed with Hinode: outflows related to a coronal mass ejection. Publ. Astron. Soc. Pac. 59, 801. DOI. ADS.

Harrison, R.A.: 1995, The nature of solar flares associated with coronal mass ejection. Astron. Astrophys. 304, 585. ADS.

Hirayama, T.: 1974, Theoretical model of flares and prominences, I: Evaporating flare model. Solar Phys. 34, 323. DOI. ADS.

Houdebine, E.R., Foing, B.H., Rodono, M.: 1990, Dynamics of flares on late-type dMe stars, I: Flare mass ejections and stellar evolution. Astron. Astrophys. 238, 249. ADS.

Houdebine, E.R., Foing, B.H., Doyle, J.G., Rodono, M.: 1993, Dynamics of flares on late-type dMe stars, 3: Kinetic energy and mass momentum budget of a flare on AD Leonis. Astron. Astrophys. 278, 109. ADS.

Hudson, H.S., Webb, D.F.: 1997, Soft X-ray signatures of coronal ejections. In: Crooker, N., Joselyn, J.A., Feynman, J. (eds.) Coronal Mass Ejections, AGU Geophysics Monograph 99, 27. DOI. ADS.

Hudson, H.S., Acton, L.W., Freeland, S.L.: 1996, A long-duration solar flare with mass ejection and global consequences. Astrophys. J. 470, 629. DOI. ADS. 
Janvier, M., Aulanier, G., Bommier, V., Schmieder, B., Démoulin, P., Pariat, E.: 2014, Electric currents in flare ribbons: observations and three-dimensional standard model. Astrophys. J. 788, 60. DOI. ADS.

Ji, H., Wang, H., Schmahl, E.J., Moon, Y.-J., Jiang, Y.: 2003, Observations of the failed eruption of a filament. Astrophys. J. Lett. 595, L135. DOI. ADS.

Joshi, A.D., Forbes, T.G., Park, S.-H., Cho, K.-S.: 2015, A trio of confined flares in AR 11087. Astrophys. J. 798, 97. DOI. ADS.

Kahler, S.W.: 1992, Solar flares and coronal mass ejections. Annu. Rev. Astron. Astrophys. 30, 113. DOI.

Kazachenko, M.D., Canfield, R.C., Longcope, D.W., Qiu, J.: 2012, Predictions of energy and helicity in four major eruptive solar flares. Solar Phys. 277, 165. DOI. ADS.

Kopp, R.A., Pneuman, G.W.: 1976, Magnetic reconnection in the corona and the loop prominence phenomenon. Solar Phys. 50, 85. DOI. ADS.

Kretzschmar, M.: 2011, The Sun as a star: Observations of white-light flares. Astron. Astrophys. 530, A84. DOI. ADS.

Kushwaha, U., Joshi, B., Veronig, A.M., Moon, Y.-J.: 2015, Large-scale contraction and subsequent disruption of coronal loops during various phases of the M6.2 flare associated with the confined flux rope eruption. Astrophys. J. 807, 101. DOI. ADS.

LaBonte, B.J., Chapman, G.A., Hudson, H.S., Willson, R.C. (eds.): 1984, Solar Irradiance Variations on Active Region Time Scales, NASA CP-2310. ADS.

Leitzinger, M., Odert, P., Greimel, R., Korhonen, H., Guenther, E.W., Hanslmeier, A., Lammer, H., Khodachenko, M.L.: 2014, A search for flares and mass ejections on young late-type stars in the open cluster Blanco-1. Mon. Not. Roy. Astron. Soc. 443, 898. DOI. ADS.

Lemen, J.R., Title, A.M., Akin, D.J., Boerner, P.F., Chou, C., Drake, J.F., Duncan, D.W., Edwards, C.G., Friedlaender, F.M., Heyman, G.F., Hurlburt, N.E., Katz, N.L., Kushner, G.D., Levay, M., Lindgren, R.W., Mathur, D.P., McFeaters, E.L., Mitchell, S., Rehse, R.A., Schrijver, C.J., Springer, L.A., Stern, R.A., Tarbell, T.D., Wuelser, J.-P., Wolfson, C.J., Yanari, C., Bookbinder, J.A., Cheimets, P.N., Caldwell, D., Deluca, E.E., Gates, R., Golub, L., Park, S., Podgorski, W.A., Bush, R.I., Scherrer, P.H., Gummin, M.A., Smith, P., Auker, G., Jerram, P., Pool, P., Soufli, R., Windt, D.L., Beardsley, S., Clapp, M., Lang, J., Waltham, N.: 2012, The Atmospheric Imaging Assembly (AIA) on the Solar Dynamics Observatory (SDO). Solar Phys. 275, 17. DOI. ADS.

Lin, R.P., Dennis, B.R., Hurford, G.J., Smith, D.M., Zehnder, A., Harvey, P.R., Curtis, D.W., Pankow, D., Turin, P., Bester, M., Csillaghy, A., Lewis, M., Madden, N., van Beek, H.F., Appleby, M., Raudorf, T., McTiernan, J., Ramaty, R., Schmahl, E., Schwartz, R., Krucker, S., Abiad, R., Quinn, T., Berg, P., Hashii, M., Sterling, R., Jackson, R., Pratt, R., Campbell, R.D., Malone, D., Landis, D., BarringtonLeigh, C.P., Slassi-Sennou, S., Cork, C., Clark, D., Amato, D., Orwig, L., Boyle, R., Banks, I.S., Shirey, K., Tolbert, A.K., Zarro, D., Snow, F., Thomsen, K., Henneck, R., McHedlishvili, A., Ming, P., Fivian, M., Jordan, J., Wanner, R., Crubb, J., Preble, J., Matranga, M., Benz, A., Hudson, H., Canfield, R.C., Holman, G.D., Crannell, C., Kosugi, T., Emslie, A.G., Vilmer, N., Brown, J.C., Johns-Krull, C., Aschwanden, M., Metcalf, T., Conway, A.: 2002, The Reuven Ramaty High-Energy Solar Spectroscopic Imager (RHESSI). Solar Phys. 210, 3. DOI. ADS.

Liu, R., Titov, V.S., Gou, T., Wang, Y., Liu, K., Wang, H.: 2014, An unorthodox X-class long-duration confined flare. Astrophys. J. 790, 8. DOI. ADS.

Maehara, H., Shibayama, T., Notsu, Y., Notsu, S., Honda, S., Nogami, D., Shibata, K.: 2015, Statistical properties of superflares on solar-type stars based on 1-min cadence data. Earth Planets Space 67, 59. DOI. ADS.

Mason, J.P., Woods, T.N., Caspi, A., Thompson, B.J., Hock, R.A.: 2014, Mechanisms and observations of coronal dimming for the 2010 August 7 event. Astrophys. J. 789, 61. DOI. ADS.

Masson, S., Antiochos, S.K., DeVore, C.R.: 2013, A model for the escape of solar-flare-accelerated particles. Astrophys. J. 771, 82. DOI. ADS.

Milligan, R.O., Dennis, B.R.: 2009, Velocity characteristics of evaporated plasma using Hinode/EUV imaging spectrometer. Astrophys. J. 699, 968. DOI. ADS.

Montes, D., Saar, S.H., Collier Cameron, A., Unruh, Y.C.: 1999, Optical and ultraviolet observations of a strong flare in the young, single K2 dwarf LQ Hya. Mon. Not. Roy. Astron. Soc. 305, 45. DOI. ADS.

Osten, R.A., Wolk, S.J.: 2015, Connecting flares and transient mass-loss events in magnetically active stars. Astrophys. J. 809, 79. http://stacks.iop.org/0004-637X/809/i=1/a=79.

Ottmann, R., Schmitt, J.H.M.M.: 1996, ROSAT observation of a giant X-ray flare on Algol: Evidence for abundance variations? Astron. Astrophys. 307, 813. ADS.

Pallavicini, R., Serio, S., Vaiana, G.S.: 1977, A survey of soft X-ray limb flare images - the relation between their structure in the corona and other physical parameters. Astrophys. J. 216, 108. DOI. ADS.

Pesnell, W.D., Thompson, B.J., Chamberlin, P.C.: 2012, The Solar Dynamics Observatory (SDO). Solar Phys. 275, 3. DOI. ADS. 
Polito, V., Reeves, K.K., Del Zanna, G., Golub, L., Mason, H.E.: 2015, Joint high temperature observation of a small C6.5 solar flare with IRIS/EIS/AIA. Astrophys. J. 803, 84. DOI. ADS.

Qiu, J., Hu, Q., Howard, T.A., Yurchyshyn, V.B.: 2007, On the magnetic flux budget in low-corona magnetic reconnection and interplanetary coronal mass ejections. Astrophys. J. 659, 758. DOI. ADS.

Reames, D.V.: 2013, The two sources of solar energetic particles. Space Sci. Rev. 175, 53. DOI. ADS.

Reinard, A.A., Biesecker, D.A.: 2008, Coronal mass ejection-associated coronal dimmings. Astrophys. J. 674, 576. DOI. ADS.

Rust, D.M., Hildner, E.: 1976, Expansion of an X-ray coronal arch into the outer corona. Solar Phys. 48, 381. DOI. ADS.

Sadykov, V.M., Vargas Dominguez, S., Kosovichev, A.G., Sharykin, I.N., Struminsky, A.B., Zimovets, I.: 2015, Properties of chromospheric evaporation and plasma dynamics of a solar flare from IRIS. Astrophys. J. 805, 167. DOI. ADS.

Sammis, I., Tang, F., Zirin, H.: 2000, The dependence of large flare occurrence on the magnetic structure of sunspots. Astrophys. J. 540, 583. DOI. ADS.

Schrijver, C.J.: 2009, Driving major solar flares and eruptions: A review. Adv. Space Res. 43, 739. DOI. ADS.

Schrijver, C.J., Beer, J.: 2014, Space weather from explosions on the Sun: how bad could it be? Eos 95, 201. DOI. ADS.

Schrijver, C.J., Beer, J., Baltensperger, U., Cliver, E.W., Güdel, M., Hudson, H.S., McCracken, K.G., Osten, R.A., Peter, T., Soderblom, D.R., Usoskin, I.G., Wolff, E.W.: 2012, Estimating the frequency of extremely energetic solar events, based on solar, stellar, lunar, and terrestrial records. J. Geophys. Res. 117, 8103. DOI. ADS.

Sterling, A.C., Hudson, H.S.: 1997, Yohkoh SXT observations of X-ray "dimming" associated with a halo coronal mass ejection. Astrophys. J. Lett. 491, L55. DOI. ADS.

Sturrock, P.A.: 1966, Model of the high-energy phase of solar flares. Nature 211, 695. DOI. ADS.

Sun, X., Bobra, M.G., Hoeksema, J.T., Liu, Y., Li, Y., Shen, C., Couvidat, S., Norton, A.A., Fisher, G.H.: 2015, Why is the great solar active region 12192 flare-rich but CME-poor? Astrophys. J. Lett. 804, L28. DOI. ADS.

Svestka, Z.: 1986, On the varieties of solar flares. In: Neidig, D.F. (ed.) The Lower Atmosphere of Solar Flares; Proceedings of the Solar Maximum Mission Symposium, National Solar Observatory, Sunspot, 332. ADS.

Thalmann, J.K., Su, Y., Temmer, M., Veronig, A.M.: 2015, The confined X-class flares of solar active region 2192. Astrophys. J. Lett. 801, L23. DOI. ADS.

Thompson, B.J., Cliver, E.W., Nitta, N., Delannée, C., Delaboudinière, J.-P.: 2000, Coronal dimmings and energetic CMEs in April-May 1998. Geophys. Res. Lett. 27, 1431. DOI. ADS.

Tian, H., Li, G., Reeves, K.K., Raymond, J.C., Guo, F., Liu, W., Chen, B., Murphy, N.A.: 2014, Imaging and spectroscopic observations of magnetic reconnection and chromospheric evaporation in a solar flare. Astrophys. J. Lett. 797, L14. DOI. ADS.

Tsuboi, Y., Koyama, K., Murakami, H., Hayashi, M., Skinner, S., Ueno, S.: 1998, ASCA detection of a superhot 100 Million K X-ray flare on the weak-lined T Tauri star V773 Tauri. Astrophys. J. 503, 894. DOI. ADS.

Tsuneta, S.: 1996, Structure and dynamics of magnetic reconnection in a solar flare. Astrophys. J. 456, 840. DOI. ADS.

White, S.M., Thomas, R.J., Schwartz, R.A.: 2005, Updated expressions for determining temperatures and emission measures from GOES soft X-ray measurements. Solar Phys. 227, 231. DOI. ADS.

Wiegelmann, T., Inhester, B.: 2010, How to deal with measurement errors and lacking data in nonlinear force-free coronal magnetic field modelling? Astron. Astrophys. 516, A107. DOI. ADS.

Woods, T.N., Eparvier, F.G., Hock, R., Jones, A.R., Woodraska, D., Judge, D., Didkovsky, L., Lean, J., Mariska, J., Warren, H., McMullin, D., Chamberlin, P., Berthiaume, G., Bailey, S., Fuller-Rowell, T., Sojka, J., Tobiska, W.K., Viereck, R.: 2012, Extreme Ultraviolet Variability Experiment (EVE) on the Solar Dynamics Observatory (SDO): Overview of science objectives, instrument design, data products, and model developments. Solar Phys. 275, 115. DOI. ADS.

Wu, S.T., de Jager, C., Dennis, B.R., Hudson, H.S., Simnett, G.M., Strong, K.T., Bentley, R.D., Bornmann, P.L., Bruner, M.E., Cargill, P.J., Crannell, C.J., Doyle, J.G., Hyder, C.L., Kopp, R.A., Lemen, J.R., Martin, S.F., Pallavicini, R., Peres, G., Serio, S., Sylwester, J., Veck, N.J.: 1986, Flare energetics. In: Kundu, M.R., Woodgate, B. (eds.) Energetic Phenomena on the Sun, Proceedings of the Solar Maximum Mission Workshop, NASA CP-2439, 5. ADS.

Yashiro, S., Gopalswamy, N., Akiyama, S., Michalek, G., Howard, R.A.: 2005, Visibility of coronal mass ejections as a function of flare location and intensity. J. Geophys. Res. 110, 12. DOI. ADS.

Zirin, H., Ingham, W., Hudson, H., McKenzie, D.: 1969, De-occultation X-ray events of 2 December, 1967. Solar Phys. 9, 269. DOI. ADS. 